\title{
VERDECKTE DISKRIMINIERUNG IN DER SYSTEMATIK DER GRUNDFREIHEITEN DER EUROPÄISCHEN GEMEINSCHAFT
}

\author{
Dr. Hacl CAN*
}

\section{ÖZET}

Avrupa Topluluğu, kendi sınırlart içerisinde malların, kişilerin, hizmetlerin ve sermayenin serbestçe dolaşabileceği bir iç pazarın kurulmasını hedeflemektedir. Bu amaçla, Topluluğun kurucu antlaşmasını teşkil eden Roma Antlaşmasında zorunlu haller dışında, üye devletler arasındaki ekonomik faaliyetleri zorlaştıran tüm engeller yasaklanmıştır. Kurucu antlaşmada pazar katılımctları arasında "tabiiyet" ve "menşe" mülahazalarıla yapılan zarar verici farklı muamelelerin engellenmesini amaçlayan ayrımcılık yasakları öngörülmüştür. İç pazar dahilinde öngörülen serbest dolaşım konularında üye devletler arasında çok büyük mesafeler kat edilmesine rağmen henüz tam bir birlik sağlanabilmiş değildir. Mamafih uygulamada üye devletler tarafindan sınır aşan pazar katılımcıların fiziki, teknik ve mali nitelikli engellerle yurtiçi rakipleri karşısında kötü duruma sokulmaları nedeniyle Topluluğun iç pazarında sınır aşan ekonomik faaliyetler sürekli olarak engellemelere maruz kalmıştır. Avrupa Toplulukları Adalet Divanı geçmişte bu konuya ilişkin olarak çok sayıda kararlar vermiş olmasına rağmen, Topluluk hukukunun uygulanması ve yorumu hâlâ ilkesel bazda ve özellikle örtülü ayrımcılığın tespiti ve nitelendirilmesinde önemli sorunlar çıkarmaktadır. Uygulamada hangi hallerin ayrımcılık teşkil ettiğini belirlemek kolay olmamaktadır. Özellikle dolaylı şekilde de olsa pazar katılımcıları arasında farklı etkiler yaratan kural ve tedbirlerin dolaşım serbestileri açısından bir ayrımcılık olarak mı, yoksa sadece basit bir sinırlama olarak görülüp görülemeyeceği konusunda tartışmalar yoğunlaşmaktadır.

Schlagwörter: Europaeische Gemeinschaft, Grundfreiheiten des Binnenmarktes, (verdeckte) Diskriminierung, Rechtfertigunggründe, EuGH

Anahtar kelimeler: Avrupa Topluluğu, İ̧ Pazarın Temel Serbestileri, (örtülü) Ayrimcılık, Hukuka Uygunluk Nedenleri, ATAD

* 9 Eylül Üniversität, Juristische Fakültät, Lehrbeauftragter 


\section{Einleitung}

Die Schaffung eines Gemeinsamen Marktes bildet den Kernpunkt der europäischen Integration und zugleich die wesentliche Grundlage der Europäischen Gemeinschaft. Diese ursprüngliche Zielsetzung des E(W)GVertrages erfuhr 1985 einen bedeutenden Wandel durch das ,Weißbuch der Kommission über die Vollendung des Binnenmarktes"1, das in der „Einheitlichen Europäischen Akte" vom 28. Februar $1986^{2}$ seine primärrechtliche Verankerung fand. Der gemeinsame Markt bzw. Binnenmarkt ${ }^{3}$ der Gemeinschaft umfaßt nunmehr einen Raum ohne Binnengrenzen, in dem der freie Verkehr von Waren, Personen, Dienstleistungen und Kapital gewährleistet ist (Art. 14 Abs. 2 EGV). Dieser läßt sich aber nicht ganz verwirklichen, wenn Marktteilnehmer anderer Mitgliedstaaten im Falle eines grenzüberschreitenden Wirtschaftsverkehrs schlechter als ihre inländischen Konkurrenten behandelt wird. Die Umsetzung bzw. Anwendung der diesbezüglichen vertraglichen Vorgaben wird jedoch nach wie vor durch eine Vielzahl unterschiedlicher nationaler Regelungen und Praktiken, die die grenzüberschreitende wirtschaftliche Betätigung entgegenstehen, verhindert.

Die Problematik der Gewährleistung der Grundfreiheiten beschäftigt Rechtsprechung und Literatur schon über Jahrzente hinweg. Der Europäische Gerichtshof (EuGH) hat sich bereits häufig mit den Grundfreiheiten des $\mathrm{E}(W) \mathrm{G}-$ Vertrages befaßt und diesbezüglich zahlreiche Urteile gefällt. Als Folge der Rechtsprechung des EuGH erzeugten die Regelungen des EG-Vertrages tiefgreifende Wirkungen in den nationalen Rechtsordnungen. Die Auslegung und Anwendung des Gemeinschaftsrechts werfen jedoch weiterhin Probleme von grundsätzlicher Bedeutung, vor allem hinsichtlich der Einordnung der verdeckten Diskriminierung, auf. Die Frage, wann eine Diskriminierung vorliegt und ob verdeckte, mittelbare unterschiedliche Auswirkungen als Diskriminierung oder als bloße Beschränkung einzuordnen sind, bereitet weiterhin erhebliche Schwierigkeiten.

Die vorliegende Arbeit setzt es sich zum Ziel, die Beeinträchtigung des Binnenmarktes durch diskriminierende Maßnahmen der Mitgliedstaaten zu analysieren, wobei zentral auf Rechtsfragen der Grundfreiheiten des Binnenmarktes eingegangen werden soll. An dieser Stelle konzentriert sich die Untersuchung grundsätzlich auf verdeckte Diskriminierung im Binnenmarkt sowie auf deren Rechtsfolgen.

\footnotetext{
Dok. KOM(85)310endg.

${ }^{2}$ Bulletin der EG Beilage Nr. 2/86. Die EEA trat am 1. Juli 1987 in Kraft.

${ }^{3}$ Umstritten ist allerdings in der Literatur, ob sich die beiden Begriffe "Gemeinsamer Markt" und

"Binnen-markt" decken oder inhaltsverschieden sind. Ausführliche Erläuterungen bei Thure Schubert, Der Gemeinsame Markt als Rechtsbegriff - Die allgemeine Wirtschaftsfreiheit des EG-Vertrages, C.H. Beck, München 1999, S. 141.
} 


\section{Diskriminierung als Grundform der Beschränkung der Grundfreiheiten $^{4}$}

\section{A. Begriff und deren Abgrenzung}

Der Begriff der "Diskriminierung" stammt aus dem lateinischen Wort "discrimen" ${ }^{\text {"5 }}$. Darunter wird heute im allgemeinen eine "benachteiligende Ungleichbehandlung" aufgrund des Vorliegens oder des Nichtvorliegens bestimmter Merkmale verstanden ${ }^{6}$. Nach ständiger Rechtsprechung des EuGH liegt eine Diskriminierung vor, wenn "vergleichbare Sachverhalte rechtlich unterschiedlich oder unterschiedliche Sachverhalte rechtlich gleich behandelt werden." ${ }^{\text {"7 }}$ Unterschiedliche Behandlung ist allerdings im negativen Sinn, d.h. zum Nachteil von bestimmten Personen oder Produkten, zu verstehen ${ }^{8}$. Die Gleichbehandlung der unterschiedlichen Sachverhalten bedeutet ebenfalls eine Diskriminierung.

Die Diskriminierung behindert ohne weiteres die Ausübung der Grundfreiheiten ${ }^{9}$ und sind deshalb im Wortlaut der Grundfreiheiten besonders deutlich angesprochen ${ }^{10}$. Sie stellt eine "Grundform der Beschränkung ${ }^{611}$ dar $^{12}$ und ist als solche, anders als die anderen Beschränkungen der Grundfreiheiten, im EG-Vertrag ausdrücklich verboten.

"Diskriminierung stellt (allerdings) nicht die einzige Form einer Beschränkung der Grundfreiheiten dar "13, ist daher gegenüber den anderen Formen der Beschränkung der Grundfreiheiten, insbesondere gegenüber der bloß unterschiedlichen Behandlung, die nicht als Benachteiligung einzustufen ist, abzugrenzen. Unter dem Begriff "Diskriminierung" ist hinsichtlich der Grundfreiheiten des Binnenmarktes die Ungleichbehandlung zum Nachteil der grenzüberschreitenden Produkte und Personen zu verstehen. Ein entsprechendes Diskriminierungsverbot gewährleistet die Gleichheit von Marktteilnehmern in

\footnotetext{
${ }_{5}^{4}$ So Hans D. Jarass, "Elemente einer Dogmatik der Grundfreiheiten II“, EuR 2000, S. 709.

5 Troberg, "Art. 52 EGV", in: Hans von der Groeben/Jochen Thiesing/Claus-Dieter Ehlermann (Hrsg.), Kommentar zum EU-/EG-Vertrag, 5. Aufl., Nomos Verl., Baden-Baden 2000, Rn. 36; Marcel Dietrich, Die Freizügigkeit der Arbeitnehmer in der Europäischen Union unter Berücksichtigung des schweizerischen Ausländerrechts, Zürich 1995, S. 381.

${ }^{6}$ Astrid Epiney, Umgekehrte Diskriminierungen, Carl Heymanns Verl., Köln, 1995, S. 3.

${ }^{7}$ EuGH, Urteil vom 3. November 1984, Rs. 283/83 (Racke), Rn. 9; Urteil vom 14. Februar 1995, Rs. C-27/93 (Schumacker), Slg. 1995, I-225, Rn. 3; vgl. auch Walter Kälini/Martina Caroni, Diskriminierungsverbot und Familiennachzug, Bern 1998, S. 16.

${ }^{8}$ Marcel Dietrich, Die Freizügigkeit der Arbeitnehmer in der Europäischen Union, unter Berücksichtigung des schweizerischen Ausländerrechts, Zürich 1995, S. 386.

${ }^{9}$ Rudolf Geiger, EUV Kommentar, 3. Aufl., 2000, Art. 28 EGV, Rn. 12.

${ }^{10}$ Jarass, S. 709 .

${ }^{11}$ EuGH, Urteil vom 3. November 1995, Rs. C-55/94 (Gebhard), Slg. 1995, I-4165, Rn. 37. Er bezeichnet die (verdeckten) Diskriminierung als Unterfall der Beschränkung. Dazu Urteil vom 3. Dezember 1974, Rs. 33/74 (van Binsbergen), Slg. 1974, 1299.

12 "Diskriminierungen als die intensivste Form der Beschränkung". So Hans-Wolfgang Arndt, Europarecht, C.F. Müller, Heidelberg 2001, S. 123.

${ }_{13}$ Jarass, S. 710.
} 
vergleichbaren Sachverhalten. Damit sollen einheitliche Wettbewerbsbedingungen innerhalb des Binnenmarktes geschaffen werden ${ }^{14}$. Ob ein Verstoß gegen die Gleichheit der Marktteilnehmer vorliegt, ist durch einen horizontalen Vergleich zwischen den ausländischen und inländischen Markteilnehmern zu ermittel $^{15}$. Die Rechtswidrigkeit einer Maßnahme hängt also nicht von der Intensität einer belastenden Anforderung, sondern von der Ungleichheit ihrer Auferlegung $a b^{16}$.

Die Beschränkung bezieht sich dagegen unabhängig von der Frage, ob eine Diskriminierung vorliegt oder nicht, indes auf die vollständigen oder teilweisen Verhinderung der Ausübung einer Grundfreiheit. Sie umfaßt zwar naturgemäß solche diskriminierenden Fälle, kann aber auch darüber hinausgehen. Beschränkungen sind unterschiedslos geltende/anwendbare, diskriminierungsfreie Maßnahmen, die die Verkehrsströme innerhalb des Binnenmarktes behindern ${ }^{17}$, z. B. Maßnahmen, die zu einer Doppelbelastung führen ${ }^{18}$. Dabei spricht der EuGH davon, daß eine Maßnahme den Gebrauch der Freiheit "weniger attraktiv mache","zusätzliche Kosten" verursache oder eine "abschreckende Wirkung" auf den Ge-brauch der Freiheit ausübe. Falls eine Regelung benachteiligende Wirkung entfaltet, stellt sie eine (verdeckte) Diskriminierung dar. Demnach verbietet ein Beschränkungsverbot nicht nur jegliche Diskriminierung, sondern auch jede innerstaatliche Maßnahme, welche die Freiheit der Marktteilnehmer unabhängig von ihren Unterscheidungsmerkmalen behindert ${ }^{19}$ (z.B., Verpackungsvorschriften für Waren, Berufsausübungsregelungen, Besteuerung von Dienstleistungen) ${ }^{20}$.

\section{B. Ausdrückliche Verankerung der Diskriminierungsverbote im EG- Vertrag}

Das Diskriminierungsverbot ist primärrechtlich ausdrücklich angeordnet. Der EG-Vertrag verbietet in seinem sachlichen Anwendungsbereich ${ }^{21}$ jede Diskriminierung und verlangt damit strikte Inländergleichbehandlung. Er enthält bezüglich des Diskriminierungsverbots neben einer allgemeinen Klausel

\footnotetext{
${ }^{14}$ Klaus Lackhoff, Die Niederlassungsfreiheit des EGV - nur ein Gleichheit oder auch ein Freiheitsrecht?, Dunckler \& Humblot Verl., Berlin 1999, S. 223.

${ }_{15}$ Thorsten Kingreen, Die Struktur der Grundfreiheiten des Europäischen Gemeinschaftsrechts, Dunckler \& Humblot, Berlin 1999, S. 39.

${ }_{17}^{16}$ Ibid.

17 Ibid.,

${ }^{18}$ Hans-Wolfgang Arndt, Ibid., S 117.

${ }^{19}$ Hierbei ist allerdings anzumerken, daß im Bereich der Verkaufsmodalitäten eine Maßnahme gleicher Wirkung nur unter diskriminierenden Beschränkungen liegt. Dazu. EuGH, Urteil vom 24. November 1993, verb. Rs. C-267-268/91 (Keck und Mithouard), Slg. 1993, I-6097; vgl. auch Margit Hintersteininger, Binnenmarkt und Diskriminierungsverbot unter besonderer Berücksichtigung der Situationen nichtstaatlicher Handlungs-einheiten, Dunckler \& Humblot, Berlin 1999, S. 22-23.

${ }^{20}$ Hintersteininger, S. 22.

${ }_{21}$ Somit sind Ungleichbehandlungen in Bereichen, die nicht im EG-Vertrag enthalten, gemeinschaftsrechtlich unerheblich.
} 
in Art. 12 EGV auch zahlreiche besondere Regelungen. Die Diskriminierungsverbote sind eine Ausformung des allgemeinen Gleichheitssatzes ${ }^{22}$.

In Art. 12 Abs. 1 EGV sind die Adressaten des Diskriminierungsverbots nicht ausdrücklich genannt. Das Diskriminierungsverbot richtet sich in erster Linie an die Mitgliedstaaten sowie die Gemeinschaftsorgane ${ }^{23}$. Die Mitgliedstaaten sollen nicht die eigenen Bürger bevorzugen dürfen. Die Gemeinschaftsorgane sind ebenfalls in ihren Handlungen dieses Verbot zu beachten. Darüber hinaus wendet sich das Diskriminierungsverbots auch an Private, soweit ihnen autonome Regelungsbefugnisse gegenüber Einzelnen zustehen (z.B. kollektive Regelung im Arbeits- und Dienstleistungsbereich, berufsportliche Regelungen eines Sportverbandes) ${ }^{24}$. Die Rechte des Einzelnen in diesen Bereichen können nämlich auch durch das Verhalten des Betroffenen beeinträchtigt werden. Nach herrschender Meinung ${ }^{25}$ entfaltet das Diskriminierungsverbot diese Drittwirkung ${ }^{26}$ im Anwendungsbereich der Verträge gegenüber jedem privaten Dritten grundsätzlich auch ohne Einschränkung.

Das allgemeine Diskriminierungsverbot des Art. 12 EGV gilt allerdings nur vorbehaltlich besonderer Bestimmungen des Vertrages. Seine selbständige Bedeutung besteht daher nur in den Anwendungsbereichen des Vertrages, die kein ausdrückliches Diskriminierungsverbot enthalten (z.B. im Bereich des Zugangs zum berufsausbildenden Unterricht) ${ }^{27}$. Es wird insbesondere in den Bestimmungen über die Grundfreiheiten des Binnenmarktes bereichsspezifisch konkretisier $^{28}$. Sie beinhalten in ihrem Kernpunkt die Diskriminierungsverbote bzw. Gleichbehandlungsgebote und verdrängen damit das allgemeine Diskriminierungsverbot ${ }^{29}$. Daraus folgt, daß auch das allgemeine Diskriminierungsverbot des Art. 12 Abs. 1 EGV nicht verletzt ist, wenn eine nationale Maßnahme nicht gegen die besonderen Diskriminierungsverbote der Grundfreiheiten verstö $\mathrm{Bt}^{30}$. Zur Auslegung der Diskriminierungsverbote kann aber doch das allgemeine Diskriminierungsverbot des Art. 12 EGV herangezogen werden ${ }^{31}$.

${ }^{22}$ EuGH, Urteil vom 1. Oktober 18, Rs. $147 / 79$ (Hochstraß), Slg. 1980, 3019.

${ }^{23}$ Vgl. Holoubek, in: Schwarze (Hrsg., EU-Kommentar, 1. Aufl., 2OOO, Art. 12 EGV Rn. 21 ff.

${ }^{24}$ EuGH, Urteil vom 12. Dezember 1974, Rs. 36/74 (Walrave), Slg. 1974, 1405; Urteil vom 15. Dezember 1995, Rs. C-415/93 (Bosman), Slg. 1995 I-4912; Geiger, EUV'/EGV Kommentar, 3. Aufl., 2000, Art. 12 EGV, Rn. 4.

${ }^{25}$ Geiger, EUV/EGV Kommentar, 3. Aufl., 2000, Art. 12 EGV, Rn. 5; von Bogdandy, Rn. 29.

${ }^{26}$ Zur Drittwirkung des Diskriminierungsverbots siehe die Rechtsprechung des EuGH, Urteil vom

12. Dezember 1974, Rs. 36/74, (Walrave und Koch), Slg. 1974, 1405, Rn. 16 (19); Urteil vom 15. Dezember 1995, Rs. C-415/93 (Bosman), Slg. 1995 I-4912, Rn. 83 ff.

${ }^{27}$ EuGH, Urteil vom 13. Februar 1985, Rs. 293/83 (Gravier), Slg. 1985, 593; Geiger, EUV/EGV Kommentar, 3. Aufl., 2000, Art. 12 EGV, Rn. 2.

${ }_{28}^{28}$ Geiger, EUV/EGV' Kommentar, 3. Aufl., 2000, Art. 10, Rn. 1; Epiney, Ibid., S. 7.

${ }^{29}$ EuGH, Urteil vom 2. Februar 1989, Rs. 186/87 (Cowan/Trésor public), Slg. 1989, 195, Rn. 14.

${ }^{30}$ Hans Georg Fischer, Europarecht, 2. Aufl., C.H. Beck, München 1997, S. 230, Rn. 10.

${ }^{31}$ Armin von Bogdandy, "Art. 6 EGV", Rn. 55 in: Eberhard Grabitzl Meinhard Hilf (Hrsg.), EUV-Kommentar. 


\section{Charakteristika der Grundfreiheiten}

Um etwas über verdeckte Diskriminierungen sagen zu können, muss man sich zuvor die Systematik der Grundfreiheiten klarmachen. Wie oben erwähnt wurde, ist der gemeinschaftliche Binnenmarkt grundsätzlich vom freien Verkehr von Waren, Personen, Dienstleistungen und Kapital geprägt. Zur Gewährleistung einer völligen Bewegungsfreiheit für Personen und Waren aller Art ist im EG-Vertrag eine Reihe von Grundfreiheiten verankert, welche sich systematisch in die vier Gruppen einteilen lassen. Sie werden als die "vier Grundfreiheiten" des EG-Vertrages genannt. Ihre wesentliche Funktion ist die Verhinderung der rechtswidrigen Beschränkungen im Binnenmarkt ${ }^{32}$.

Die Grundfreiheiten gewährleisten jeweils bestimmte Tätigkeiten mit grenzüberschreitenden Elementen ${ }^{33}$ und bezwecken somit eine entsprechende Öffnung der nationalen Märkte, wobei die Mitgliedstaaten dazu verpflichtet sind, in ihrer Rechtsordnung alle Hemmnisse in wirtschaftlicher Art, die das ordnungsgemäße Funktionieren des Gemeinsamen Marktes beeinträchtigen, zu beseitigen und keine neuen Beschränkungen einzuführen. Sie begründen dem Einzelnen subjektive Rechte, die aufgrund ihrer unmittelbaren Wirkung vor nationalen Gerichten und bei Behörden geltend gemacht werden können ${ }^{34}$. Im Falle des Verstoßes einer nationalen Rechtsnorm gegen eine Grundfreiheit des Binnenmarktes kann daher verlangt werden, sie nicht anzuwenden ${ }^{35}$. Die Grundfreiheiten entfalten aber auch eine Drittwirkung und schützen insofern vor Beschränkungen, die von Privaten ausgehen.

Die Freiheit des Warenverkehrs (Art. 23 bis 31 EGV) sowie des Kapitalund Zahlungsverkehrs (Art. 56 bis $60 \mathrm{EGV}$ ) gewährleistet in erster Linie einen umfassenden Schutz vor sachlich nicht gerechtfertigten Beschränkungen. Hinsichtlich dieser Grundfreiheiten steht das Verbot der ungerechtfertigten Beschränkung im Vordergrund. Sie sind im EG-Vertrag ausdrücklich als Beschränkungsverbot formuliert. Demgemäß kommt in den Art. 28-30 sowie Art. 56 und Art $58 \mathrm{EGV}$ das Wort "Beschränkungen" häufig vor". Die Beschränkungen können aber auch diskriminierende Belastungen umfassen ${ }^{37}$. Da diskriminierende Maßnahmen ohne weiteres freien Verkehr von Waren und Kapital behindern ${ }^{38}$, sind der freie Warenverkehr und der freie Kapital- und Zahlungsverkehr auch als ein Diskriminierungsverbot einzuordnen, obgleich die

\footnotetext{
${ }^{32}$ Wolfgang Arndt, S. 115

${ }^{33}$ Jarass, S. 710 .

${ }^{34}$ Dirk Ehlers, "Die Grundfreiheiten des europäischen Gemeinschaftsrechts (Teil I)", JURA 2001, S. 267.

${ }_{35}^{35}$ Wolfgang Arndt, S. 112

${ }^{36}$ Marcus Heintzen, Europarecht II, Vorlesung vom 19.5.1998, Freie Universität Berlin, Fachbereich Rechts-wissenschaft SS 1998.

${ }_{37}$ Ehlers, S. 269; Fischer, Georg, S. 239, Rn. 26.

${ }^{38}$ Gert Nicolaysen, Europarecht II: Das Wirtschaftsrecht im Binnenmarkt, Nomos Verl., Baden-Baden 1996, S. 45.
} 
diesbezüglichen Vorschriften des EG-Vertrages wörtlich weniger deutlich auf eine Diskriminierung hinweisen ${ }^{39}$ als die der anderen Grundfreiheiten ${ }^{40}$.

Die in Art. 39, Art. 43 und Art. 50 EGV geregelten Personenverkehrsfreiheiten (die Arbeitnehmerfreizügigkeit, die Niederlassung- und Dienstleistungsfreiheit) sind hingegen im Text des EG-Vertrages explizit als Diskriminierungsverbot formuliert und knüpfen unmittelbar an die Staatsangehörigkeit" an (Art. 39 Abs. 2 EGV: "Abschaffung jeder auf der Staatsangehörigkeit beruhenden unterschiedlichen Behandlung ${ }^{642}$; Art. 43 Abs.2 EGV: "die Aufnahme und die Ausübung von Tätigkeiten nach den Bestimmungen des Aufnahmestaats für seine eigene Angehörigen"; Art. 50 Abs. 3 EGV: "unter den Voraussetzungen, welche dieser Staat für seine eigenen Angehörigen vorschreibt" $)$. Sie statuieren vor allem ein Gleichheitsrecht und konkretisieren damit das allgemeine Diskriminierungsverbot des Art. 12 EGV. Umstritten ist hier, ob dem Wortlaut dieser Vorschriften zu entnehmen ist, ob diese Grundfreiheiten auch solche staatlichen Maßnahmen verbieten können, die unterschiedslos In- und Ausländer in gleicher Weise belasten. Inzwischen ist vom $\mathrm{EuGH}^{43}$ und der überwiegenden Lehre ${ }^{44}$ anerkannt worden, daß die Personenverkehrsfreiheiten über das Diskriminierungsverbot hinaus auch ein Beschränkungsverbot beinhalten ${ }^{45}$. Zur Begründung seiner Auffassung weis der Gerichtshof zum einen darauf hin, daß Art. 3 Abs. 1 lit. c) EGV die Beseitigung von "Hindernissen" des grenzüberschreitenden Waren-, Personen- und Dienstleistungsverkehrs vorsieht. Markthindernisse können auch nichtdiskriminierender Natur sein ${ }^{46}$. Ohne Beschränkungsverbote werde der Schutz der Grundfreiheiten erheblich erschwert, was gegen den effet utile Grundsatz verstoßen würde. Die Wahrnehmung der Grundfreiheiten kann nicht nur durch Diskriminierungen, sondern auch in gleicher Weise durch unterschiedslos wirkende Maßnahmen verhindert werden. Zum anderen sei ein

\footnotetext{
${ }^{39}$ Zulässige Beschränkungen dürfen keineswegs ein Mittel der "willkürlichen Diskriminierungen" sein (Art. 30 und Art. 58 Abs. 3 EGV).

${ }^{40}$ Rudolf Streinz, Europarecht, 5. Aufl., Springer Verl., Berlin 2001, S. 269, Rn. 667; Jarass, Ibid., S. 709.

${ }^{41}$ Der persönliche Anwendungsbereich beschränkt sich nicht auf natürliche Personen, sondern erstreckt sich auch auf juristische Personen. Vgl. EuGH, Urteil vom 20. Oktober 1993, verb. Rs. C-92/92 und C-326/92 (Phil Collins), Slg. 1993, I-5145, Rn. 30.

${ }^{42}$ V gl. Art. 1-9 VO 1612/68/EGW (ABl, 1968 Nr. L 257/2).

${ }^{43}$ EuGH, Urteil vom 15. Dezember 1995, Rs. C-415/93 (Bosman), Slg. 1995 I-4912; Urteil vom 12. Februar 1974, Rs. 152/73 (Sotgiu), Slg. 1974, 153; Urteil vom 15. Oktober 1969, Rs. 15/69 (Südmilch), Slg. 1969, 363; zur Niederlassungsfreiheit vgl. Urteil vom 21. Juni 1974; Rs. 2/74 (Reyners), Slg. 1974, 631; Urteil vom 28. April 1977, Rs. 71/1976 (Thieffry), Slg. 1977, 765; Urteil vom 30. Dezember 1995, Rs. C-55/94 (Gebhard), Slg. 1995, I-4165; zur Dienstleistungsfreiheit vgl. Urteil vom 3. Dezember 1974, Rs. 33/1974 (van Binsbergen), Slg. 1974, 1299; Urteil vom 24. März 1994, Rs. C-275/92 (Schindler), Slg. 1992, I-1039.

${ }_{4}$ Zur Überblick siehe Thorsten Kingreen, Ibid, S. 38 ff.; Dagmar Schiek, Europäisches Arbeitsrecht, 1. Auf1., 1997, S. 109 ff; Rudolf Streinz, Ibid., S. 270, Rn. 671; Nicolaysen, S. 165 f.

${ }^{45}$ Rudolf Streinz, S. 270, Rn. 671; Michael Schweitzer/Waldemar Hummer, Europarecht, 5. Aufl., Frankfurt am Main 1996, S.330, Rn. 1075.

${ }_{46}$ Bröhmer, "Art. 43 EGV", in: Jürgen Schwarze (Hrsg.), EU-Kommentar, 1. Aufl., 2000, Nomos Verl., Baden-Baden, Rn. 18.
} 
allgemeines Diskriminierungsverbot schon in Art. 12 EGV verankert. In diesem Zusammenhang enthalten die Grundfreiheiten nur eine deklatorische und konkretisierende Aussage. Um den Grundfreiheiten eine über Art. 12 EGV hinausgehende eigenständige Bedeutung zu verleihen, seien daher grundsätzlich auch unterschiedslos anwendbare Regelungen unter das Beschränkungsverbot $\mathrm{zu}$ subsumieren ${ }^{47}$. So müssen sich auch unterschiedslos auf Inländer und Ausländer anwendbare Vorschriften bzw. Maßnahmen auf ihre Vereinbarkeit mit dem Gemeinschaftsrecht am Maßstab der Verhältnismäßigkeit rechtfertigen lassen.

Zusammenfassend ist folgendes festzuhalten: Die Grundfreiheiten sind zwar im EG-Vertrag unterschiedlich ausgestaltet ${ }^{48}$, weisen aber hinsichtlich ihrer Gewährleistungen sowie ihrer Eingriffsrechtfertigung Parallelitäten auf ${ }^{49}$. Die Freiheiten des Waren-, Kapital- und des Zahlungsverkehrs sind zunächst als Beschränkungsverbote zu verstehen; aber dabei sind die Diskriminierungsverbote nicht ausgeschlossen. Denn Schutz gegen Diskriminierungen ist in Art. 30 und Art. 59 EGV gewährleistet. Der Wortlaut der Vorschriften der Personenverkehrsfreiheiten (Art. 39, Art. 43 und Art. 50 EGV) legt an sich ein Diskriminierungsverbot viel näher als der der Vorschriften des freien Warenverkehrs (Art. 28 und Art. 30 EGV) sowie des freien Kapital- und Zahlungsverkehrs (Art. 56 ff. EGV) ${ }^{50}$. Deren Anwendungsbereich wurde vom EuGH jedoch zu Beschränkungsverboten ausgeweitet ${ }^{51}$. Sie gehen in ihren Anwendungsbereichen über ein Gleichheitsrecht hinaus und gebieten nunmehr nicht nur den Abbau diskriminierender, sondern jeglicher den grenzüberschreitenden freien Verkehr ungerechtfertigt behindernder, hemmender oder beschränkender nationaler Maßnahmen ${ }^{52}$.

Die sämtlichen Grundfreiheiten gewährleisten also trotz ihrer unterschiedlichen Ausgestaltung je nach Fallkonstellation entweder die Gleichheit der Marktteilnehmer oder ihre Freiheit gegenüber dem Mitgliedstaat.

\footnotetext{
${ }^{47}$ Wolfgang Arndt, S. 116.

48 Götz-Sebastian Hök, Einführung in das Recht der Europäischen Union und die Grundfreiheiten in der Europäischen Union, 1998, S. 5.

${ }^{49}$ Wolfgang Weis, "Nationales Steuerrecht und Niederlassungsfreiheit: Von der Konvergenz der Grundfreiheiten als Beschränkungsverbote zur Auflösung der Differenzierung zwischen unterschiedslosen und unterschiedlichen Maßnahmen“, EuZW 1999, S. 496.

${ }_{50}$ Peter Behrens, "Die Konvergenz der wirtschaftlichen Freiheiten im Europäischen Gemeinschaftsrecht" "EuR 1992, S. 150.

${ }_{51}$ Für die Warenverkehrsfreiheit: EuGH, Rs 8/74 (Dassonville), Slg 1974, 837/852;, Rs 120/78 (Rewe), 649, 662 Rn 8; Dienstleistungsfreiheit: Rs 33/74 (van Binsbergen), Slg 1974, 1291/1310; Arbeitnehmerfreizügigkeit: Rs C-415/93 (Bosman), Slg 1995, I-4921.

52 Franz-Josef Schöne, "Die 'umgekehrte Diskriminierung' im EWG-Vertrag nach der Rechtsprechung des Europäischen Gerichtshofs - Dargestellt am Beispiel der Dienstleistungsfreiheit nach Art. 59 ff. EWG-Vertrag“, RIW 1989, 450.
} 
Die beiden Funktionen der Grundfreiheiten können sogar kumulativ zur Anwendung kommen ${ }^{53}$.

\section{I. Allgemeine Tatbestandsmerkmale der Beschränkung der Grundfreiheiten in diskriminierender Art}

\section{A. Grenzüberschreitender Sachverhalt}

Die Grundfreiheiten gewährleisten nicht auf vollständige Marktfreiheit bzw. -gleichheit im Binnenmark, sondern beschränken sich im wesentlichen nur auf den Marktzugang von Personen, Waren sowie Kapital und verpflichten insofern die Mitgliedstaaten dazu, alle Hindernisse (darunter auch Diskriminierungen) zu beseitigen ${ }^{54}$. Die Diskriminierungsverbote ent-falten nur im Rahmen des Anwendungsbereiches des EG-Vertrages Rechtswirkungen ${ }^{55}$. In den Anwendungsbereich des Vertrages fallen wiederum alle die Grundfreiheiten betreffenden Regelungen. Da die Grundfreiheiten tatbestandlich an grenzüberschreitende Sachverhalte anknüpfen ${ }^{56}$, beschränken sich die Diskriminierungsverbote ebenfalls nur auf grenzüberschreitende Sachverhalte ${ }^{57}$ und gewährleisten in diesem Rahmen die Inländergleichbehandlung ${ }^{58}$. Die Ungleichbehandlungen von Sachverhalten, die mit den Grundfreiheiten zusammenhängen, müssen also einen gearteten Bezug zu einem anderen Mitgliedstaat aufweisen ${ }^{59}$. Daher können insbesondere rein innerstaatliche Sachverhalte der Mitgliedstaaten nicht an den Grundfreiheiten der Gemeinschaft gemessen werden. Die Anwendung des Gemeinschaftsrechts kann jedoch umgekehrt auch zu einer Inländerdiskriminierung (d.h. Schlechterstellung von Inländern gegenüber Angehörigen anderer Mitgliedstaaten) führen. Eine solche Schlechterstellung entsteht insbesondere dadurch, daß die Angehörigen eines Mitgliedstaates den Erfordernissen des innerstaatlichen Rechts nachkommen müssen, während Angehörige an-derer Mitgliedstaaten (aber auch Inländer, welche die vertraglichen Rechte in Anspruch genommen haben) aufgrund des Gemeinschaftsrechts von diesen Erfordernissen freigestellt sind ${ }^{60}$. Der EuGH betrachtet in seiner ständigen Rechtsprechung ${ }^{61}$ solche Schlechterstellung von Inländern als eine reine interne Angelegenheit des jeweiligen Mitgliedstaates, auf die das Diskriminierungsverbot des EG-Vertrages nicht anwendbar ist ${ }^{62}$. Die

\footnotetext{
${ }^{53}$ Streinz, S. 271, Rn. 672.

${ }^{54}$ Ehlers, S. 269.

${ }_{56}^{55}$ EuGH, Urteil vom 13. Februar 1985, Rs. 293/83 (Gravier), Slg. 1985, 593.

${ }_{57}^{56}$ Streinz, S. 276, Rn. 683.

57 Die Abgrenzung zwischen inländischen und grenzüberschreitenden Sachverhalten kann allerdings im Einzel-fall schwierig sein. Kingreen, S. $140 \mathrm{ff}$.

${ }_{58}^{58}$ Ehlers, S. 269.

${ }^{59}$ Hintersteininger, S. 24.

${ }^{60}$ EuGH, Urteil vom 12. März 1987, Rs. 178/84 (Reinheitsgebot), Slg. 1987, 1227; Urteil vom 27. Oktober 1982

${ }_{61}$ EuGH, Urteil vom 12. Juli 1984, Rs. $107 / 83$ (Klopp), Slg. 1984, 2971.

${ }^{62}$ Ehlers, S. 269.
} 
Anwendung der nationalen Maßnahmen darf jedoch den Wettbewerb der nationalen Märkte nicht beeinträchtigen ${ }^{63}$. Das Diskriminierungsverbot erstreckt sich ausnahmsweise nur dann auf inländische Marktteilnehmer, wenn der Anwendungsbereich des EG-Vertrages den betreffenden Sachverhalt erfaßt ${ }^{64}$. Darüber hinaus können sich Marktteilnehmer von Drittstaaten sowie Staatenlose insofern auf Diskriminierungsverbot berufen, als sie in den Anwendungsbereich des EG-Vertrages einbezogen $\operatorname{sind}^{65}$.

\section{B. Ungleichbehandlung zum Nachteil der grenzüberschreitenden Produkte und Personen}

Jedes Diskriminierungsverbot setzt eine Differenzierung bzw. unterschiedliche Behandlung voraus ${ }^{66}$. Hinsichtlich der Grundfreiheiten fallen jedoch nicht alle Ungleichbehandlungen unter den Anwendungsbereich des Diskriminierungsverbots. Gemeinschaftsrechtlich verboten sind nur die Diskriminierungen aus Gründen der "Andersstaatlichkeit $t^{667}$ von Marktteilnehmern. Erfaßt werden dabei nur solche nationale Regelungen, die nach der Herkunft von Personen und Produkten differenzieren ${ }^{68}$. Eine Diskriminierung ist in diesem Sinne dann gegeben, wenn eine nationale Maßnahme im Bereich des freien Personenverkehrs, nämlich der Freizügigkeit der Arbeitnehmer, der Niederlassungsfreiheit oder Dienstleistungsfreiheit, an die Staatsangehörigkeit oder an die (ausländische) Ansässigkeit ${ }^{99}$ anknüpft ${ }^{70}$. Im Bereich des freien Warenverkehrs sowie des freien Kapital- und Zahlungsverkehrs liegt eine Diskriminierung vor, wenn eine Maßnahme zum Nachteil grenzüberschreitender Waren, Kapital oder Zahlungen führt. "Im Rahmen der Warenverkehrs- und Dienstleistungsfreiheit ist aber grundsätzlich auch die Anknüpfung an die Staatsangehörigkeit des Herstellers der Produkte denkbar." 71

Die Diskriminierung kann nicht nur das Gesetz selbst, sondern auch durch Verwaltungspraktiken erfolgen ${ }^{72}$. Das Ausmaß der Diskriminierung ist nicht

\footnotetext{
${ }^{63}$ EuGH, Urteil vom 7. Mai 1997, verb. Rs.C-321, 322, 323, 324/94 (Pistre), Slg. 1997, I-2343, Rn. $41 \mathrm{ff}$.

${ }^{64}$ So sind z.B. gemeinschaftsrechtliche Bezüge vorhanden, wenn ein Inländer eine Ware in einen anderen Mitgliedstaat ausführen will oder in einem anderen Mitgliedstaat eine berufliche Qualifikation erwerben und sie danach in ihrem Heimatstaat anerkennen lassen will. Zur Ubersicht der Rechtsprechung des EuGH siehe Geiger, EUV/EGV Kommentar, 3. Aufl., 2000, Art. 12 EGV, Rn. 2.

${ }_{65}$ Manfred Zuleeg, "Art. 6 EGV", in: Hans von der Groeben/Joechen Thiesing/Claus-Dieter Ehlermann, (Hrsg.), Kommentar zum EU-/EG-Vertrag, 5. Aufl., Nomos Verl., Baden-Baden 2000, Rn. 16.

${ }_{66}^{6}$ Rn. 19.

${ }^{67}$ Hans Peter Ipsen, Europäisches Gemeinschaftsrecht, Tübingen 1972, S. 600.

${ }_{68}^{68}$ Margit Hintersteininger, S. 24.

${ }^{69}$ So wurde etwa im Urteil vom 17. Dezember 1981, Rs. (Webb), Slg. 81, 3305, Rn. 14; vgl. auch Urteil vom 3. Dezember 1974, Rs. 33/74 (van Binsbergen), Slg. 1974, 1299, Rn. 10/12.

${ }_{70}$ Jarass, S. 710.

${ }_{72}^{71}$ Hintersteininger, S. 24.

${ }^{72}$ Nicolaysen, S. 47.
} 
relevant. Auch die geringfügigen Verstöße sind verboten, weil der Einzelne auch dadurch verunsichert werden kann. Die Grundfreiheiten schützen jedoch nur vor Ungleichbehandlungen. In diesem Sinne liegt daher keine Diskriminierung vor, wenn die Regelung eines Mitgliedstaates von der eines anderen Mitgliedstaates abweicht, sofern sie sich ohne Rücksicht auf die Herkunft von Marktteilnehmern auf alle Marktteilnehmer auswirken ${ }^{73}$. Dieser Freiraum der Mitgliedstaaten ist aber erheblich begrenzt, weil sie dabei das Herkunftsstaatsprinzip zu beachten haben, wonach die Vorschriften eines Bestimmungsstaats hinsichtlich der grenzüberschreitenden Marktteilnehmer zurücktreten sind, die den Vorschriften eines Herkunftsstaats begnügen ${ }^{74}$. Dies ist z.B. dann der Fall, wenn alle Prüfungen und Kontrollen bereits im Herkunftsstaat unter gleichen Voraussetzungen und Bedingungen wie im Bestimmungsstaat vorgenommen und von den entsprechenden Stellen ausgestellt worden, und daher rechtmäßig zum Verkehr zugelassen sind.

Der EuGH legt den Diskriminierungsbegriff sehr weit aus und mißt dabei einer Benachteilungsabsicht des Mitgliedstaats keine Bedeutung bei. Die Grundfreiheiten des Binnenmarktes verbieten nach der ständigen Rechtsprechung des EuGH alle Formen der Diskriminierung ${ }^{75}$. Erfaßt werden zunächst offene Diskriminierungen, die unmittelbar an die Unterscheidungsmerkmale anknüpfen. Der EuGH kennt daneben aber auch die sogenannte verdeckte oder mittelbare Diskriminierung an, die bei der Anwendung anderer Unterscheidungsmerkmale tatsächlich $\mathrm{zu}$ demselben Ergebnis führt ${ }^{76}$. In der Entscheidung Sotgiu ${ }^{77}$ hat er zur Begründung angeführt, daß dies geboten sei, um die Wirksamkeit eines der Grundprinzipien des Gemeinschaftsrechts zu wahren (effet utile) ${ }^{78}$. Ansonsten bestehe die Gefahr darin, daß das Verbot (offener) Diskriminierung leicht umgegangen werden könnte ${ }^{79}$.

Die beiden Formen der Diskriminierung werden in der Rechtsprechung und in der Literatur verschiedenartig bezeichnet ${ }^{80}$. Zur Unterscheidung der

\footnotetext{
${ }^{73}$ Ulrich Fastenrath/Maike Müller-Gerbes, Europarecht, 1. Aufl., Nomos Verl., Baden-Baden 2000, S. 66, Rn. 109; Matthias Rossi, "Das Diskriminierungsverbot nach Art. 12 EGV", EuR 2000,210.

${ }_{74}$ EuGH, Urteil vom 12. März 1987, Rs. 178/84 (Reinheitsgebot), Slg. 1987, 1227.

${ }^{75}$ EuGH, Urteil vom 13. Juli 1993, Rs. C-330/91 (Commerzbank), Slg. 1993, I-4017; vgl. auch die Richtlinie der Kommission 70/50/EWG vom 22. Dezember 1969 über die Beseitigung von Maßnahmen gleicher Wirkung wie mengenmäßige Einfuhrbeschränkungen (ABl. 1970 Nr. L 13, 29).

${ }^{76}$ Zur Arbeitnehmerfreizügigkeit siehe EuGH. Urteil vom 12. Februar 1974, Rs. 152/73 (Sotgiu), Slg. 1974, 153, Rn. 5; vgl. auch Urteil vom 30. Mai 1989, Rs. 33/88 (Allué u.a.), Slg. 1989, 1591, Rn. 11; Rs. 22/80 (Boussac/Gerstenmeier), Slg. 1980 3427; zur Niederlassungsfreiheit siehe Urteil vom 28. April 1977, Rs. 71/76, Slg. 1977, 765, zur Dienstleistungsfreiheit siehe Urteil vom 3. Februar 1982, Rs. 62/81 (Seco), Slg. 1982, 222; Urteil vom 10. Februar 1994, Rs. C-398/92 (Mund \& Fester), Slg. 1994, I-497.

7 EuGH, Urteil vom 12. Februar 1974, Rs. 152/73 (Sotgiu), Slg. 1974, 153.

${ }^{78}$ Epiney, Rn. 15.

${ }^{79}$ Lackhoff, S. 226.

${ }^{80}$ Dietrich, S. 389; Hintersteininger, S. 26.
} 
beiden Formen der Diskriminierung wird vom EuGH eine Vielzahl von Ausdrücken verwendet. Hierbei handelt es sich zumeist um folgende Bezeichnungen: offenbare und versteckte Diskriminierung; unmittelbare und mittelbare Diskriminierung, formelle und materielle Diskriminierung, oder auch direkte und indirekte Diskriminierung ${ }^{81}$. "Die terminologische Vielfalt bedeutet aber nicht unbedingt einen Unterschied in der Sache ${ }^{482}$ Sie werden im Unterschied zu einander synonym verwendet ${ }^{83}$.

\section{Einordnung der verdeckten Diskriminierung}

\section{A. Kennzeichnen und Feststellung der verdeckten Diskriminierung}

Die Abgrenzung zwischen den offenen und verdeckten Diskriminierungen hat eine große Bedeutung, weil sie sich hinsichtlich ihrer Anforderungen an eine Rechtfertigung unterscheiden. Der wesentliche Unterschied zwischen offenen und verdeckten Diskriminierungen besteht darin, daß die offenen Diskriminierungen eindeutig feststellbar sind, während die verdeckten Diskriminierungen erst durch eine zweistufige Interessenabwägung im Einzelfall festzustellen $\operatorname{sind}^{84}$. Unproblematisch ist die Feststellung einer offenen Diskriminierung, die ohne weiteres an das verbotene Differenzierungsmerkmal (bzw. Unterscheidung von Produkten und Personen nach ihrer Herkunft) anknüpft ${ }^{85}$. Der Begriff der offenen Diskriminierung meint ausdrückliche Ungleichbehandlungen wegen der Staatsangehörigkeit zum Nachteil des grenzüberschreitenden Produkt und Personenverkehrs. Im Fall einer offenen Diskriminierung wirkt sich die Differenzierung zwischen inländischen und grenzüberschreitenden Sachverhalten zum Nachteil der die Grenze überschreitenden Produkte oder Personen unmittelbar aus der getroffenen Maßnahme aus. Offene Diskriminierungen kommen in der Praxis ganz selten vor ${ }^{86}$.

Die Feststellung einer verdeckten Diskriminierung ist dagegen äußerst schwierig. Unklar ist hier vor allem, wann eine unterschiedslos anwendbare Maßnahme zu einem vergleichen Ergebnis führt wie bei einer offenen Diskriminierung $^{87}$. Der EuGH hat diese Problematik vor allem im Zusammenhang mit der Diskriminierung aus Gründen der Staatsangehörigkeit behandelt. Eine gemeinschaftsrechtswidrige Diskriminierung ist nach dieser

\footnotetext{
${ }^{81}$ Zuleeg, Rn. 4.

${ }_{83}^{82}$ Kingreen, S. 39; Armin von Bogdany, Ibid., Rn. 12 ff.

${ }^{83}$ Josef Schöne, S. 450.

${ }^{84}$ Dietrich, S. 38.

${ }_{85}$ EuGH, Urteil vom 14. Februar 1995, Rs. C-279/93 (Schumacker), Slg. 1995, I-225, Rn. 30; Matthias Herdegen, Europarecht, 2001, C.H. Beck, München 2001, S. 74, Rn. 97.; Schlag, "Art. 43 EGV“ in: Jürgen Schwarze (Hrsg.), EUV Kommentar, 1. Aufl., Nomos Verl., Baden Baden, 2000, Rn. 34.

${ }^{86}$ Kingreen, S. 38.

${ }^{87}$ Lackhoff, S. 229.
} 
Rechtsprechung des EuGH unabhängig davon, ob eine Diskriminierung auf eine ausdrücklich nach der Staatsangehörigkeit oder nach anderem Kriterium differenzierende Regelung zurückgeht. Entscheidend dafür ist vielmehr, daß im Ergebnis eine mit der Staatsangehörigkeit zusammenhängende Diskriminierung bewirkt wird. In diesem Sinne stellt eine solche nationale Regelung, die verlangt, daß die Geschäftsführer und Direktoren aller Sicherheitsunternehmen im Inland wohnen müssen, eine verdeckte Diskriminierung dar ${ }^{88}$.

Nach der ständigen Rechtsprechung des EuGH ist ein typisches Charakteristikum der verdeckten Diskriminierung die Anknüpfung differenzierender Maßnahmen an ein anderes, scheinbar neutrales und unterschiedslos für in und ausländische Marktteilnehmer geltendes Merkmal ${ }^{89}$, wobei jedoch letztlich das gleiche Regelungsergebnis wie bei Heranziehung des offenen Differenzierungsmerkmals, nämlich Diskriminierung von grenzüberschreitenden Produkten, Personen oder Kapital, erzielt wird ${ }^{90}$. Denn diese scheinbar neutralen Anforderungen können eben von inländischen Marktteilnehmern ohne weiteres automatisch (oder leichter) erfüllt werden, bedeuten aber für ausländische Marktteilnehmer in der Regel ein erhebliches Hindernis $^{91}$. Sie gelten zwar unterschiedslos, führen aber faktisch zu einer Benachteiligung von grenzüberschreitenden Produkten bzw. Personen ${ }^{92}$. Sie können die verlangten Merkmale praktisch oder nur unter erschwerten Bedingungen erfüllen. Die einseitig belastende Wirkung wird hier also "auf Umwegen" ${ }^{63}$ herbeigeführt. In diesem Sinne liegt z.B. eine Diskriminierung vor, wenn ein Mitgliedstaat den Anspruch eines Ausländers von einer bestimmten Art von Aufenthaltstitel (vom Besitz einer Aufenthaltsberechtigung oder Aufenthaltserlaubnis) abhängig macht, während Inländer nur ihren Wohnsitz in diesem Staat haben müssen ${ }^{94}$. Das Vorliegen einer Diskriminierung wird in solchen Fällen also nicht nach formalen, sondern nach materiellen Gesichtspunkten ermittelt ${ }^{95}$.

Eine verdeckte Diskriminierung ergibt sich nicht explizit aus dem Wortlaut der Norm oder aus der Praktika, sondern erst aus der Analyse ihrer materiellen

\footnotetext{
${ }^{88}$ EuGH, Urteil vom 25. Juli 1991, Rs. C-2721/89 (Factortame II), Slg. 1991, I-3905, Rn. 32.

${ }^{89}$ Hintersteininger, S. 35; Lackhoff, S. 227.

${ }^{90}$ EuGH, Urteil vom 29. Oktober 1980, Rs. 22/80 (Boussac), Slg. 1980 3427; Urteil vom 10. Februar 1994, Rs. C-398/92 (Mund \& Fester/Hatrex), Slg. 1994, I-467; Urteil vom 12. Februar 1974, Rs. 152/73 (Sotgiu), Slg. 1974, 164; Urteil vom 10. März 1993, Rs. C111/91(Kommission/Luxemburg), Slg. 1993, I-840; Urteil vom 23. Januar 1997, Rs. C-29/95 (Pastoors), Slg. 1997, I-285); Urteil vom 27. Januar 2000, Rs. C-190/98 (Graf), Slg. 2000, I-0001, Rn. 14.

${ }_{91}^{91}$ Hintersteininger, S. 35; Bröhmer, Ibid., Rn. 19.

${ }_{93}^{2}$ Kingreen, S. 38.

93 Wolfgang Kilian, Europäisches Wirtschaftsrecht, C.H. Beck, München, 1996, S. 128, Rn. 316.

${ }_{94}$ EuGH, Urteil vom 4. Mai 1999, Rs. C-262/96 (Sürül), Rn. 103 und 105; Rs. 33/88 (allué und Coonan), Slg. 1989, 1591; Fastenrath/Müller-Gerbes, Europarecht, 1. Aufl., 2000, S. 66 Rn. 108.

${ }^{95}$ Kingreen, S. 39.
} 
Wirkungen ${ }^{96}$. Z.B. gilt eine Regelung zwar sowohl für eigene als auch für fremde Marktteilnehmer, kann sich aber unterschiedlich auswirken ${ }^{97}$. Dabei ist unerheblich, ob die Regelung nur Marktteilnehmer eines oder aller Mitgliedstaaten benachteiligt oder sogar mit Vorteilen für Marktteilnehmer bestimmter Mitgliedstaaten einhergeht ${ }^{98}$.

Eine Benachteiligungsabsicht ist keine Voraussetzung für das Vorliegen einer verdeckten Diskriminierung ${ }^{99}$. Für die Annahme einer verdeckten Diskriminierung reicht eine rein kausale Herbeiführung einer vergleichbaren Situation zu einer offenen Diskriminierung durch eine unterschiedslos anwendbare Maßnahme aus ${ }^{100}$. Eine verdeckte Diskriminierung liegt aber auch dann vor, wenn lediglich bezweckt wird, daß ungleiche Bedingungen für die grenzüberschreitenden Produkte und Personen geschaffen werden ${ }^{101}$.

Eine verdeckte Diskriminierung setzt allerdings nach der ständigen Rechtssprechung des EuGH ein "quantitatives Element"102 voraus, wonach die grenzüberschreitenden Produkte oder Personen die Anforderung der nationalen Regelung "meist" "häufig" "“typischerweise" nicht oder schwer erfüllen ${ }^{103}$. Der EuGH erachtet grundsätzlich jedes Kriterium für geeignet, eine (verdeckte) Diskriminierung herbeizuführen, solange es "im wesentlichen","in erster Linie" ""typischer" oder "hauptsächlich" eine Benachteiligung der ausländischen Marktteilnehmer bewirkt ${ }^{104}$. Die verdeckte Diskriminierung muß nicht unbedingt dieselbe, sondern nur lediglich die gleiche Wirkung wie eine offene Diskriminierung entfalten ${ }^{105}$. Ein eindeutiges Kriterium dafür, wann eine Differenzierung gleiche Auswirkungen auf die grenzüberschreitenden Produkte oder Personen hat, besteht nicht. Dies ist nicht allgemeingültig zu beantworten, sondern vielmehr im jeweiligen Einzelfall zu untersuchen ${ }^{106}$.

\section{A. Typische Beispiele der verdeckten Diskriminierung in den Grundfreiheiten der Gemeinschaft}

Verdeckte Diskriminierungen lassen sich nicht abschließend bestimmen, sondern vielmehr nur durch die Ermittlung von Umständen des jeweiligen

\footnotetext{
${ }^{96}$ Kingreen, S. 39; . Dietrich, S. 393; Rossi, S. 211.

${ }^{97}$ EuGH, Urteil vom 23. Mai 1996, Rs. C-237/94 (O’Flynn), Slg. 1996, 2617.

${ }_{98}$ EuGH, Rs 20/85 (Roviello), Slg 1988, 2805, 2852, Rn 16.

99 Gundel, Jörg, "Die Rechtfertigung von faktisch diskriminierenden Eingriffen in die Grundfreiheiten des EGV“", JURA 2001, 79 f.; Kingreen, S 39.

${ }_{100}$ Vgl. Art. 3 Abs. 1 VO 1612/68; Lackhoff, S. 227; Epiney, S. 101.

${ }^{101}$ Dietrich, S. 397.

${ }^{102}$ Lackhoff, S. 230

${ }^{103}$ Vgl. EuGH, Urteil vom 28. April 1977, Rs. $71 / 76$ (Thieffry), Slg. 1977, 765; Urteil vom 7. Juli 1988, Rs. 143/87 (Stanton), Slg. 1988, 3877; Urteil vom 25. Juli 1991, Rs. C-221/89 (Factortame II), Slg. 1991, I-3905, vgl. Dietrich, S. 395; anders jedoch Astrid Epiney, S. 105 ff.

${ }_{104}$ Hintersteininger, S. 37.

${ }^{105}$ Dietrich, S. 395.

${ }^{106}$ Ibid.,
} 
Einzelfalls feststellen. Als Beispiele verdeckter Diskriminierungen lassen sich aus der Rechtsprechung des EuGH etwa anführen:

Hinsichtlich des freien Warenverkehrs stellen spezielle Etikettierungs- und Verpackungsanforderungen usw. typische Fälle verdeckter Diskriminierung dar ${ }^{107}$, weil ein Unternehmen seine Produkte für den Export anders als den heimischen Markt etikettieren muß, so daß in diesen Fällen der wirtschaftliche Anpassungszwang höhere Kosten verursacht, was die Wettbewerbsfähigkeit des Unternehmens beeinträchtigt und die erwünschte grenzüberschreitende Betätigung erschwert, nämlich die Produktion verteuert und eventuell erschwert.

Hinsichtlich der Arbeitnehmerfreizügigkeit wirken vor allem Ansässigkeits ${ }^{108}$ und Spracherfordernisse verdeckt diskriminierend, weil es sich hauptsächlich zum Nachteil der Angehörigen anderer Mitgliedstaaten auswirkt, da Gebietsfremde meist Ausländer sind ${ }^{109}$. Demnach ist eine verdeckte Diskriminierung dann der Fall ${ }^{110}$, wenn eine Differenzierung beruht etwa

- auf der Staatsangehörigkeit der Familienangehörigen (bzw. Ehepartner, Kinder),

- auf dem Wohnsitz und Arbeitsort der Arbeitnehmer oder denjenigen der Familienange-hörigen,

- auf einer bestimmten Mindestaufenthaltsdauer im Inland,

- auf dem Sitzland der besuchten Ausbildungseinrichtung,

- auf der Art einer Arbeitstätigkeit sowie auf das Land früher erworbenen Berufserfahrung,

- auf Arbeitgeberbeiträgen zur Sozialversicherung im Tätigkeitsstaat, obwohl im Heimatstaat gleichwertige Abgaben geleistet werden, und die Arbeitnehmer damit keinen Anspruch auf Leistungen aus der Sozialversicherung im Tätigkeitsstaat erwerben,

\footnotetext{
${ }^{107}$ EuGH, Urteil vom 12. März 1987, Rs. 178/84 (Reinheitsgebot), Slg. 1987, 1227; Urteil vom 14. Juli 1988, Rs. 407/85 (Drei Glocken), Slg. 1988, 4233.

${ }^{108}$ EuGH Rs 152/73 (Sotgiu), Slg 1974, 153, Rn 11; Rs C-272/92 (Spotti), Slg 1993, 5185, Rn 18; Rs C-266/95 (Garcia), Slg, 1997, I-3279.

${ }_{109}$ EuGH, Rs C-350/96 (Clean Car Autoservice), Slg 1998, I-2547 Rn 29.

110 Zur Übersicht der Rechtsprechung des EuGH siehe Troberg, "Art. 52 EGV", in: Hans von der Groe-ben/Joechen Thiesing/Claus-Dieter Ehlermann (Hrsg.), Kommentar zum EU-/EGVertrag, 5. Aufl., Nomos Verl., Baden-Baden 2000 , Rn. 40 ff.; Dietrich, S. 393-394.
} 
Besonderheiten gelten bei der Niederlassungsfreiheit für die Anknüpfung an den Sitz der Gesellschaft ${ }^{111}$ (Art. 48 Abs. 1 EGV) Hinsichtlich der Niederlassungsfreiheit handelt sich etwa um folgende verdeckte Diskriminierungen ${ }^{112}$;

- Erteilung öffentlicher Aufträge nur an Unternehmen, bei denen die öffentliche Hand eine Mehrheitsbeteiligung hält,

- Zahlung eines Zuschlags zu einer Rückzahlung nicht geschuldeter Steuern nur zugunsten der Gesellschaften mit steuerlichem Sitz im Inland,

- nur Gesellschaften mit Hauptgeschäftssitz im Inland als Wertpapiermakler,

- höherer Einkommensteuersatz für bestimmte Gebietsfremde, soweit nicht zur Gewährleistung der steuerrechtlichen Kohärenz der Steuerregelung des betreffenden Mitgliedstaats erforderlich.

Hinsichtlich der Dienstleistungsfreiheit sind folgende Fälle als verdeckte Diskriminierung einzuordnen ${ }^{113}$ :

- Erfordernis eines Wohnsitzes im Inland für einen Versicherungsmakler;

- sonstige unterschiedliche Rechtsfolgenanknüpfung je nach Wohnsitz des Betroffenen, z.B. die Versagung des Lohnsteuerausgleichs für nicht Gebietansässige,

- die Kontingentierung von Bootsanlegeplätzen für Bootseigentümer mit Wohnsitz in einem anderen Mitgliedstaat,

- unterschiedliche Tarife für gleichwertige Lotsentätigkeit je nach Zulassung zur Seekabotage, weil nur unter inländischer Flagge fahrende Schiffe zur Kabatoge zugelassen sind und diese in der Regel von Inländischen Wirtschaftstreibenden betrieben werden,

- ein Vorbehalt von EDV Dientsleistungen für mehrheitlich im Besitz der öffentlichen Hand befindliche Unternehmen,

111 EuGH, Rs C-212/97 (Centros), Slg 1999, I-1459, 1491, Rn 20; verb Rs C-4 u. 5/95 (stöber), Slg 1997, I-511, 546 Rn 38.

${ }_{112}$ Zur Übersicht der Rechtsprechung des EuGH siehe Holoubek, "Art. 49 EGV“, in: Jürgen Schwarze (Hrsg.), EU-Kommentar, Npmos Verl., Baden-Baden, 1. Aufl., 2000, Rn. 76 f.; Lackhoff, S. 232 ff.

${ }^{113}$ Kluth, "Art. 50 EGV“", in: Jürgen Schwarze (Hrsg.), EUV-Kommentar, Nomos Verl., BadenBaden, 1. Aufl. 2000, , Rn. 56: Geiger, Art. 39, Rn. 15. 
- Vermutungsregelungen über die steuerliche Nichtabzugsfähigkeit von Aufwendungen für im Ausland abgehaltene Fortbildungsveranstaltungen.

\section{Rechtfertigung der verdeckten Diskriminierung}

\section{A. Allgemeines}

Ist eine Maßnahme als Diskriminierung im Anwendungsbereich einer Grundfreiheit einzustufen, stellt sie sich nicht unbedingt als eine Verletzung der Grundfreiheit dar. Vielmehr kann sie unter bestimmten Voraussetzungen erlaubt sein. Diese kann allerdings erst dann der Fall sein, wenn eine geeignete Grundlage vorhanden ist.

\section{B. Grundlagen der Rechtfertigung}

\section{Ausdrücklich im EG-Vertrag geregelte Schranken der Grundfreiheiten}

Der EG-Vertrag legt dazu über die Bereichsausnahmen der Grundfreiheiten ${ }^{114}$ hinaus ausdrücklich eine Reihe von Rechtfertigungstatbeständen fest. Gemäß Art. 30 EGV stehen die Regelungen der Art. 28 und 29 den Ein-, Aus- und Durchfuhrverboten oder beschränkungen nicht entgegen, die aus Gründen der öffentlichen Sittlichkeit, Ordnung und Sicherheit, zum Schutz der Gesundheit und des Lebens von Menschen, Tieren oder Pflanzen, des nationalen Kulturguts, künstlerischen, geschichtlichen oder archäologischen Werts oder des gewerblichen und kommerziellen Eigentums, gerechtfertigt sind, soweit in der Maßnahme nicht ein Mittel zur willkürlichen Diskriminierung oder eine verschleierte Beschränkung des freien Warenverkehrs liegt ${ }^{115}$. Die Freiheit des Personenverkehrs steht Beschränkungen aus Gründen der öffentlichen Ordnung, Sicherheit und Gesundheit nicht entgegen (Art. 39 Abs. 3, Art. 46 Abs. 1 und Art. 55 EGV) ${ }^{116}$. Für die Kapitalverkehrsfreiheit behält Art. 58 EGV das Recht der Mitgliedstaaten vor, die einschlägigen Vorschriften

\footnotetext{
${ }^{114}$ Der EG-Vertrag sieht eine Reihe von Bereichsausnahmen der Grundfreiheiten vor. Laut Art. 39 Abs. 4 EGV findet die Freizügigkeit der Arbeitnehmer keine Anwendung auf die Beschäftigung in der öffentlichen Ver-waltung. Die Niederlassungs- und Dienstleistungsfreiheit findet keine Anwendung auf Tätigkeiten, die in einem anderen Mitgliedstaat dauernd oder zeitweise mit der Ausübung der öffentlicher Gewalt verbunden sind (Art. 45 und 55 EGV). Hinsichtlich des freien Warenverkehrs hat der EuGH die Verkaufsmodalitäten vom Beschränkungsverbot ausgenommen. Vgl. EuGH, Urteil vom 17. Dezember 1980, Rs. 149/79 (Kommission/Belgien), Slg. 1980, 3881; Urteil vom 21. Juni 1974, Rs. $2 / 74$ (Reyners), Slg. 1974, 631, Urteil vom 13. Juli 1993, Rs. C-42/93 (Thijssen), Sig. 1993, I-4047.

${ }_{115}$ EuGH, Urteil vom 12. März 1987, Rs. 178/84 (Reinheitsgebot), Slg. 1987, 1227; Urteil vom 31. Januar 1984, Rs. 40/82 (Kommission/Vereinigtes Königreich), Slg. 1984, 283; Urteil vom 14. Dezember 1979, Rs. 34/79 (Henn und Darby), Slg. 1979, 3795; Urteil vom 14. Juli 1981, Rs. 187/80 (Merck/Stephar), Slg. 1981, 2063.

116 EuGH, Urteil vom 4. Dezember 1974, Rs. 41/1974 (van Duyn), Slg. 1974, 1337; Urteil vom 28. Oktober 1975, Rs. 36/1975 (Rutili), Slg. 1975, 1219; Urteil vom 15. März 1988, Rs. 147/86 (Kommission/Griechenland), Slg. 1988, 1637.
} 
ihres Steuerrechts anzuwenden, die Steuerpflichtige mit unterschiedlichem Wohnort oder Kapitalanlageort unterschiedlich behandeln, und die unerläßlichen Maßnahmen zu treffen, um Zuwiderhandlungen gegen innerstaatliche Rechts- und Verwaltungsvorschriften, insbesondere auf dem Gebiet des Steuerrechts und der Aufsicht der Finanzinstitute, zu verhindern, sowie Meldeverfahren für den Kapitalverkehr zwecks administrativer oder statistischer Information vorzusehen oder Maßnahmen zu ergreifen, die aus Gründen der öffentlichen Ordnung und Sicherheit gerechtfertigt sind.

Alle benannten Rechtfertigungsgründe sind auf alle Arten von Beschränkungen (sowohl diskriminierender als auch nichtdiskriminierender Art) anwendbar ${ }^{117}$. Wie dem Wortlaut dieser Vorschriften zu entnehmen ist, handelt es sich dabei um die strikte Aufzählung von Zielen nichtwirtschaftlicher $\mathrm{Art}^{118}$. Derartige Bestimmungen sind eine Ausnahmeregelung und daher eng auszulegen ${ }^{119}$. Sie gelten folglich nicht für den Fall, daß es sich nur um einen Vorwand handelt und der Staat in Wirklichkeit andere Ziele verfolgt, insbesondere wirtschaftlicher Art oder nur die Absicht hat, die Verwaltungstätigkeit zu erleichtern ${ }^{120}$. Ebenfalls werden die Interessen dritter Staaten oder einer Völkergemeinschaft nicht berücksichtigt. Die Ausnahmeregelungen beziehen sich nämlich nur auf reine nationale Interessen der Mitgliedstaaten.

Die Auslegung der Rechtsnormvorbehälte und der Rechtsfertigungsgründe kann zwar die Mitgliedstaaten nach ihren eigenen Maßstäben vornehmen. Die Schutzmaßnahmen dürfen jedoch nicht der Umgehung der Grundfreiheiten dienen $^{121}$. Die im EG-Vertrag genannten Rechtfertigungsgründe greifen nämlich nur dann ein, wenn eine tatsächliche und hinreichend schwere Gefährdung vorliegt, die ein Grundinteresse der Gesellschaft berührt ${ }^{122}$. Nationale Maßnahmen müssen zum Schutz der öffentlichen Interessen unbedingt notwendig und gerechtfertigt sein ${ }^{123}$. Die Beschränkung der Grundfreiheit muß zudem angemessen sein. Es dürfen keine anderen effektiven Mittel möglich sein, die die Ausübung der Grundfreiheiten weniger stark beschränken.

\footnotetext{
${ }^{117}$ Jarass, S. 717.

${ }^{118}$ EuGH, Urteil vom 17. Juli 1981, Rs. 113/80 (Irische Souvenirs), Slg. 1981, $1625 \mathrm{ff}$.

119 EuGH, Urteil vom 17. Juli 1981, Rs. 113/80 (Irische Souvenirs), Slg. 1981, 1625; Urteil vom 10. Dezember 1968, Rs. 7/68 (Kunstschätze I), Slg. 1968, 633.

120 EuGH, Urteil vom 9. Juni 1982, Rs. 95/81 (Kommission/Italien), Slg. 1982, 2187 ff.; Urteil vom 15. Juli 1982, Rs. 40/82 (Kommission/Vereinigtes Königreich), Slg. 1982, 2793; Urteil vom 24. Juni 1971, Rs. 57/0 (van Eick/Kommission), Slg. 1971, 613.

${ }_{121}$ EuGH, Urteil vom 15. Januar 1982, Rs. 40/82 (Kommission/Vereinigtes Königreich), Slg. 1982, 2826; Urteil vom 16. Dezember 1980, Rs. 271/80 (Fietje), Slg. 1980, 3855.

${ }_{122}$ EuGH, Urteil vom 29. Oktober 1998, Rs. C-114/97 (Kommission/Spanien), Rn. 46; Urteil vom 11. März 1986, Rs. 121/8 (Connegate, Slg. 1986, 1007 ff.

${ }_{123}$ EuGH Urteil vom 15.März 1988, Rs. $147 / 86$ (Kommission/Griechenland), Slg. 1988, 1637, Rn. 7; Urteil vom 21. Juni 1974, Rs. C-2/74 (Reyners), Slg. 1974, 631.
} 


\section{Vom EuGH entwickelte Schranken der Grundfreiheiten}

\section{a. Zwingende Erfordernisse des Allgemeininteresses}

Der EuGH relativiert den Anwendungsbereich der Beschränkungsverbote der Grundfreiheiten, indem er unter bestimmten Bedingungen den Mitgliedstaaten gestattet, nationale Hemmnisse beizubehalten. Die Erweiterung der Rechtfertigungsmöglichkeiten geht Hand in Hand mit der Entwicklung der Grundfreiheiten zu Beschränkungsverboten ${ }^{124}$. Der EuGH kennt seit seiner "Cassis de Dijon" Entscheidung ${ }^{125}$ neben den ausdrücklichen Rechtfertigungsgründen des EG-Vertrages (Art. 30, 39 Abs. 3, 46 Abs. 1, ggf. i. V. m. 55, 58 Abs. 1) ausnahmsweise weitere Schranken für Eingriffe der Mitgliedstaaten in die Grundfreiheiten an. Nach der "Cassis-Formel" des EuGH müssen auch solche Handelshemmnisse hingenommen werden, die aus den Unterschieden der autonomen nationalen Regelungen ergeben, soweit sie notwendig sind, um "zwingenden Erfordernissen des Gemeinwohls", insbesondere einer wirksamen steuerlichen Kontrolle, des Schutzes der öffentlichen Gesundheit, der Lauterkeit des Handelsverkehrs des Umweltschutzes $^{126}$, des Verbraucherschutzes ${ }^{127}$ und der Aufrechterhaltung der Medienvielfalt ${ }^{128}$ gerecht $\mathrm{zu}$ werden ${ }^{129}$. Die Rechtfertigung durch zwingende Erfordernisse kann allerdings nur in den bereits nicht harmonisierten Bereichen in Betracht kommen ${ }^{130}$. Der neue Begriff der "zwingenden Erfordernisse" wurde in seiner ständigen Rechtsprechung weiter entwickelt ${ }^{131}$. Der Katalog der "zwingenden Erfordernisse" ist nicht abschließend ${ }^{132}$, beschränkt sich jedoch auf Erfordernisse nichtwirtschaftlicher Art $^{133}$.

\footnotetext{
${ }_{124}^{124}$ Weiß, S. 493, 498; Streinz, Rn 699; Gundel, S. 79, 83.

${ }^{125}$ EuGH, Urteil vom 20. Februar 1979, Rs.120/78 (Cassis de Dijon), Slg. 1979, 649 ff.

${ }^{126}$ EuGH, Urteil vom 20. September 1988, Rs. 302/1986 (Kommission/Dänemark), Slg. 1988, 4607.

${ }^{127}$ EuGH, Urteil vom 12. März 1987, Rs. 178/1984 (Reinheitsgebot), Slg. 1987, 1227.

${ }^{128}$ EuGH, Rs C-368/95, Slg 1997, I-3689, 3715 Rn 18.

129 EuGH, Urteil vom 30 November 1995, Rs. C-55/94 (Gebhard), Slg. 1995, I-4165, Rn. 37; Urteil vom 31. März 1993, Rs. C-19/92 (Kraus), Slg. 1993, I-1663, Rn. 32, Urteil vom 15. Dezember 1995, Rs. C-415/93 (Bosman), Slg. 1995, I-4921, Rn. 104.

${ }_{130}$ Z.B. Britische Ausfuhrbeschränkungen im Verhältnis zu Spanien aus Gründen des Tierschutzes (EuGH, Urteil vom 23. Mai 1996, Rs. C-5/94 (Hedley Lomas), Slg. 1996, I-2553, Rn. 18 ff.

${ }_{131}$ Vgl. in diesem Zusammenhang EuGH, Urteil vom 20. Februar 1979, Rs.120/78 (Cassis de Dijon), Slg. 1979, 649 ff.; Rs. 229/83 (Leclerc), Slg. 1985, 1; Urteil vom 11. Juli 1985, Rs. 60 und 61/84 (Cinéthèque), Slg. 1985, 2605; Urteil vom 26. Juli 1997, Urteil vom 14. Juli 1988, Rs. C368/95 (Vereinigte Familiapress/Bauer Verlag), Slg. 1987, I-3689; Urteil vom 14. Juli 1988, Rs. 407/85 (3 Glocken), Slg. 1988, 4233; Urteil vom 9. Juli 1992, Rs. C-2/90 (Abfallimport), Slg. 1992, I-4431, Rn. 33 ff.

${ }_{132} \mathrm{Im}$ einzelnen siehe Müller-Graff, in: G/T/E (Hrsg.), Kommentar zum EU-/EG-Vertrag, 5. Aufl., 1997, Art. 30 EGV, Rn. 203 ff.

${ }_{133}$ Peter-Christian Müller-Graff, "Art. 30 EGV" in: Hans von der Groeben/Joechen Thiesing/Claus-Dieter Ehlermann (Hrsg.), Kommentar zum EU-/EG-Vertrag, 5. Aufl., Nomos Verl., Baden-Baden 2000, Rn. 204.
} 
Dem Erfordernis weiterer Schranken der Grundfreiheiten legt im wesentlichen zugrunde, daß durch die äußerst restriktive Auslegung der Ausnahmen durch die EuGH der flexiblen Anwendung der Rechtfertigungsgründe erheblich eingeschränkt ist ${ }^{134}$. Zur Kompensation der weiten Auslegung des Begriffs "Maßnahmen gleicher Wirkung" erachtet der Gerichtshof es daher für notwendig, weitere Schranken anzuerkennen.

Die dogmatische Einordnung der vom EuGH entwickelten Schranken "zwingende Erfordernisse" des Allgemeininteresses ist bisher nicht vollständig geklärt worden ${ }^{135}$. Darin sah der EuGH in seiner früheren Rechtsprechung ${ }^{136}$ immanente Tatbestandsausnahmen. In jüngeren Entscheidungen ${ }^{137}$ hat er allerdings seine Auffassung geändert und sie als Rechtfertigungsgründe bezeichnet.

Die Cassis-Formel ${ }^{138}$ richtete sich in der ursprünglichen Rechtsprechung des EuGH ausschließlich auf den freien Warenverkehr. Der EuGH hat diese Rechtsprechung allerdings in der Folgezeit auf die anderen Grundfreiheiten übertragen ${ }^{139}$. So finden heute im Anwendungsbereich der sämtlichen Grundfreiheiten neben den Rechtfertigungsgründen des EG-Vertrages die ungeschriebenen zwingenden Erfordernisse des Allgemeinwohls Anwendung. Dabei gelten die gleichen Bedingungen, die für die Warenverkehrsfreiheit im Rahmen der Cassis-Formel anzustellen sind. Der nationalen Regelung muß also ein zwingendes Erfordernis des Allgemeininteresses zu Grunde liegen. So dann ist die Verhältnismäßigkeit zu prüfen.

Hinsichtlich der ungeschriebenen Rechtfertigungsgründe für die staatlichen Eingriffe in die Grundfreiheiten hat der EuGH in seiner ständigen Rechtsprechung zwar keine einheitliche Terminologie verwendet ${ }^{140}$. Die terminologische Vielfalt führt aber nicht unbedingt zu einem Unterschied in der Sache $^{141}$,.

\footnotetext{
${ }^{134}$ Streinz, S. 282, Rn. 701.

135 Jarass, S. 719 f.

${ }^{136}$ EuGH, Rs 113/80 (Kommission/Irland), Slg 1981, 1625, Rn. 7-9.

${ }^{137}$ EuGH, Urteil vom 15. Dezember 1995, Rs. C-415/93 (Bosman), Slg. 1995, I-4921; Urteil vom 9. Juli 1997, Rs. C-368/95, Slg. 1997, I-3689.

${ }_{138}$ Ausführliche Erläuterung der Cassis-de-Dijon- Rechtsprechung des EuGH bei Peter-Christian Graff, Rn. 186 ff.

${ }^{139}$ Für die Dienstleistungsfreiheit z.B. EuGH, verb. Rs. 10 und 14/78 (van Mesemael), Slg. 1979, 35, Rn. 28; Ur-teil vom 25. Juli 1991, Rs. C-76/90 (Säger/Dennemeyer), Slg. 1991, I-4221, 542, Rn. 15; für die Nieder-lassungsfreiheit z.B. Urteil vom 11. Mai 1999, Rs. C-255/97 (Pfeiffer/Löwa), Slg. 1999, I-2835, Rn. 19; für die Arbeitnehmerfreizügigkeit z.B. Urteil vom 23. Mai 1996, Rs. C-231/94 (O'Flynn), Slg. 1996, I-2617, Rn. 19; Urteil vom 7. Mai 1998, Rs. C350/96 (Clean Car Autoservice), Slg. 1998, I-2521, 601, Rn. 31.

${ }_{140}$ Warenverkehrsfreiheit: "zwingende Erfordernisse", Arbeitnehmerfreizügigkeit: "objektive Erwägungen", Dienstleistungs- und Niederlassungsfreiheit: "zwingende Gründe des Allgemeininteresses".

${ }^{141}$ Gundel, S. 79.
} 


\section{b. Anwendung der zwingenden Erfordernisse des Allgemeinin- teresses auf die verdeckte Diskriminierung}

Die Cassis-Rechtsprechung ist vom EuGH ursprünglich ausschließlich auf alle Marktteilnehmer gleichermaßen erfassenden Maßnahmen bzw. unterschiedslos anwendbaren Beschränkungen der Grundfreiheiten angewandt worden ${ }^{142}$. Das hat zur Folge, daß bei unterschiedslos anwendbaren Maßnahmen als Rechtfertigung prinzipiell alle denkbaren sachlichen Gründe heranzuziehen sind, während die Rechtfertigung unterschiedlicher Maßnahmen nur im Rahmen der im EG-Vertrag genannten Rechtfertigungsgründe zulässig sein kann ${ }^{143}$.

Die Abgrenzung verdeckter Diskriminierungen zu den unterschiedslos anwendbaren nationalen Regelungen wird bisher uneinheitlich vorgenommen ${ }^{144}$. Dies ist besonders hinsichtlich der Rechtfertigungsgründe von erheblicher Bedeutung, weil die Rechtfertigung verdeckter Diskriminierungen genauso wie offene Diskriminierungen nur in einem beschränkten Umfang zulässig sind, nämlich nach dem EG-Vertrag festgelegten Rechtsfertigungsgründen (im Rahmen des Warenverkehrs nach Art. 30 EGV; im Rahmen der Niederlassungsund Dienstleistungsfreiheit nach Art. 46 i.V.m. Art. 55 EGV und im Rahmen der Freizügigkeit der Arbeitnehmer nach Art. 39 Abs. 3 EGV) ${ }^{145}$. Strittig ist nach wie vor, ob die ungeschriebenen Rechtsfertigunggründe darüber hinaus auch verdeckte oder gar offene Diskriminierungen zu rechtfertigen vermögen. Diese Frage hat nach der Entscheidung in der Sache Keck an Bedeu-tung gewonnen, weil sie alle vertriebsbezogenen Regelungen betrifft, die ausnahmsweise in den Anwendungsbereich des Art. 28 fallen $^{146}$.

\section{i. Meinungsstand}

\section{aa. Rechtsprechung}

Hinsichtlich der Rechtfertigung verdeckter Diskriminierung hat der EuGH in der ständigen Rechtsprechung keine klare Linie gezogen. Der EuGH schließt nämlich die Anwendung der ungeschriebenen Rechtsfertigungsgründe auf verdeckte Diskriminierung nicht aus. In jüngster Zeit hat der EuGH eine Vielzahl von Urteilen ${ }^{147}$ gefällt, die diese strikte Differenzierung zwischen

\footnotetext{
${ }^{142}$ EuGH, Rs. 177/83 (Kohl/Ringelhan), Slg. 1984, 3651, Rn. 15 und 19.

143 Vgl. EuGH, Rs. C-55/94 (Gebhard), Slg. 1995, I-1931; Rs. C-224/97 (Ciola), Slg. 1999, I2517 .

${ }_{144}^{2517}$ Jarass, S. 710

${ }^{145}$ Weiß, S. 496.

${ }^{146}$ Becker, "Art. 30 EGV", in: Jürgen Schwarze (Hrsg.), EU-Kommentar, Npmos Verl,, BadenBaden, 1. Aufl., 2000, Rn 41.

${ }_{147}$ EuGH, Urteil vom 3. Dezember 1974, Rs. 33/74 (van Binsbergen), Slg. 1974, 1299; Rs. (Kommission/Belgien), Slg. 1992, I-4431; Urteil vom 28. April 1998, Rs. C-158/96 (Kohll), Slg. 1998, I-1931; Urteil 16. Dezember 1992, Rs. C-211/91 (Kommission/Belgien), Slg. 1992, I-6757; Urteil vom 4. Mai 1993, Rs. C-17/92Rs.. (Distribuidores Cinematograficos), Slg. 1993, I-2239; Urteil vom 28. April 1998, Rs.C-120/95 (Decker), Slg. 1998, I-1831.
} 
unterschiedlichen und unterschiedslosen Maßnahmen nicht beachten ${ }^{148}$, und die Rechtfertigung diskriminierender Regelungen am Maßstab der zwingenden Erfordernisse des Allgemeinwohls geprüft, ohne allerdings auf die damit verbundene Problematik einzugehen ${ }^{149}$. Die unterschiedliche Rechtfertigungssystematik bei unterschiedlichen und unterschiedslosen Maßnahmen ist somit teilweise obsolet geworden ${ }^{150}$.

\section{bb. Literatur}

In der Literatur ist derzeit der Anwendungsbereich der zwingenden Erfordernisse des Allgemeininteresses äußerst umstritten. Hierbei werden insgesamt drei Auffassung vertreten. Ein Teil der Literatur ${ }^{151}$ ist in der Meinung, daß der EuGH die Differenzierung zwischen unterschiedlich und unterschiedslos anwendbaren Maßnahmen aufgegeben hat und stattdessen den Anwendungsbereich der ungeschriebenen Gründe auf sämtliche Diskriminierungen ausgedehnt hat ${ }^{152}$. Die Anhänger dieser Ansicht sehen generell das Beschränkungsverbot als Prüfungsmaßstab und die "zwingenden Erfordernisse" als einheitlichen Rechtfertigungsmaßstab an. Sie hat für sich eine Vereinfachung der Prüfung, außerdem wird die schwierige Unterscheidung zwischen verdeckten Diskriminierungen und Beschränkungen ${ }^{153}$ obsolet.

Die herrschende Auffassung geht hingegen von einer Unterscheidung des Anwendungsbereichs von geschriebenen und ungeschriebenen Rechtfertigungsgründen aus. An dieser Stelle ist allerdings wiederum umstritten, wo die Grenzen zwischen den Anwendungsbereichen von geschriebenen und ungeschriebenen Rechtfertigungsgründen zu ziehen sind. Im Streit geht es darum, wie die diskriminierenden Beschränkungen definiert

\footnotetext{
${ }^{148}$ Weiß, S. 497.

149 Jarass, S. 719.

${ }^{150}$ Weiß, S. 497.

${ }^{151}$ Wolfgang Weis, Ibid., 493; Astrid Epiney, "Art. 28 EGV“, in: Christian Callies/Matthias Ruffert (Hrgs,), Kommentar zu EU-Vertrag und EG-Vertrag, 1. Aufl., Luchterhand, Neuwied 1999, Rn. 38; Hakenberg, "Art. 49/50 EGV", in: Otto Lenz (Hrsg.), EGV-Kommentar, 2. Aufl.,1999, Rn. 26; Leible, "Art. 28 EGV" in: Eberhard Grabitz/Meinhard Hilf (Hrsg.), Kommentar zur Europäischen Union: Das Recht der Europäischen Union, C.H. Beck, Loseblatt, Stand Mai 2001, Rn. 20; Jarass, S. 719.

152 Im Fall des wallonischen Einfuhrverbotes für Abfälle hat der EuGH eine offene Diskriminierung mit Umweltschutzgründen gerechtfertigt. Der EuGH umging es, das Tabu einer ungeschriebenen Zulassung von formalen Diskriminierungen anzutasten, indem er der Regelung ihren diskriminierenden Charakter unter Hinweis auf das Ursprungsprinzip (Art. 174 II 2) abgesprochen hat In der Entscheidung De Agostini verweist der EuGH darauf, dass ein diskriminierendes Werbeverbot auch durch die zwingenden Gründe des Allgemeininteresses zu rechtfertigen sei. In der Rechtsprechung Svensson ging es um eine soziale Beihilfe zum Wohnungsbau, die nur dann gewährt wurde, wenn das entsprechende Baudarlehen bei einer inländischen Bank aufgenommen worden war. Zur Rechtfertigung dieser verdeckten Diskriminierung hat der EuGH zwar formell auf Art. 55 i. V. m. Art. 46 abgestellt, inhaltlich jedoch die einschlägige Rechtsprechung zur Rechtfertigung von Beschränkungen angeführt. Dazu EuGH, Rs C-2/90 (Kommission/Belgien), Slg 1992, I-4431, Rn 34; Rs C-415/93 (Bosman), Slg 1995, I-4921 Rn 129.

${ }^{153}$ Vgl. GA Tesauro, Slg 1998, I-1834, 1863 - Decker u. Kohll.
} 
werden sollen. Ein Teil der Literatur ${ }^{154}$ geht von einem absoluten Diskriminierungsverbot aus und unterscheidet infolgedessen zwischen den formalen bzw. verdeckten Diskriminierungen und den nichtdiskriminierenden Beschränkungen der Grundfreiheiten. Die Anhänger dieser Auffassung kennen lediglich hinsichtlich der nichtdiskriminierenden Beschränkungen die Möglichkeit einer Rechtfertigung durch zwingende Erfordernisse des Allgemeininteresses an. Danach seien die Diskriminierungen nur mit den ausdrücklich im EG-Vertrag genannten Gründen zu rechtfertigen, während die vollständig diskriminierungsfreien Beschränkungen zusätzlich durch die von der EuGH entwickelten ungeschriebenen Rechtsfertigungsgründe, die zwingenden Erfor-dernisse des Allgemeininteresses, gerechtfertigt werden könnten. Abgeleitet wird dies aus der Rechtsprechung des EuGH: So konstatiert der Gerichtshof in der Entscheidung Aragonesa ${ }^{155}$, dass von einem zwingenden Erfordernis nur gesprochen werden könne, wenn die Maßnahme unterschiedslos auf inländische und eingeführte Ware anwendbar sei. In der ein Werbeverbot für ausländische Arzneimittel behandelnden Entscheidung Ortscheit ${ }^{156}$ sieht der Gerichtshof das Werbeverbot als durch Art. 30 gerechtfertigt an. In der Entscheidung van Adverteerders ${ }^{157}$ heißt es, daß innerstaatliche Vorschriften, "die nicht unterschiedslos auf alle Dienstleistungen ohne Rücksicht auf deren Ursprung anwendbar und die somit diskriminierend sind" nur durch geschriebene Rechtfertigungsgründe gerechtfertigt werden können.

Der Literatur läßt sich eine weitere Auffassung entnehmen. Nach dieser Auffassung sollten zumindest die verdeckten Diskriminierungen ebenfalls wie die vollständig unter-schiedslos wirkenden Beschränkungen auch durch die zwingenden Erfordernisse des Allgemeininteresses gerechtfertigt werden ${ }^{158}$. Verdeckte Diskriminierungen seien von Beschränkungen nicht abzugrenzen und die Ausdehnung des Verbots der Diskriminierung auf verdeckte Diskrimnierungen sei überflüssig, wenn die Grundfreiheiten Beschränkungsverbote seien ${ }^{159}$. Müller-Graff ${ }^{160}$ modifiziert diese Auffassung, indem er von einem Finalitätskriterium ausgeht. Demnach ist die Berufung auf "zwingende Erfordernisse" nur dann wegen verdeckter Diskriminierung zu verwehren, wenn die Ungleichbehandlung bezweckt ist bzw. ein plausibler Zweck überhaupt nicht feststellbar ist.

154 Schneider/Wunderlich, "Art. 3 EGV", in: Jürgen Schwarze (Hrsg.), EUV-Kommentar, Nomos Verl., Baden-Baden, 1. Aufl., 2000, Rn. 37; Schweitzer/Hummer, Rn. 1139; Günter Hirsch, "Die aktuelle Rechtsprechung des EuGHH zur Warenverkehrsfreiheit", ZEuS 1999, 510 f.; Lackhoff, S. $236 \mathrm{f}$.

155 EuGH, Rs C-1/90 u. 176/90 (Aragonesa), Slg 1991, I-4151, 4184, Rn 13.

${ }_{157}^{156}$ EuGH, Slg 1994, $5243,5263 \mathrm{ff}, \mathrm{Rn} 13$.

${ }_{158}^{15} \mathrm{EuGH}$, Slg $1988,2085,2134$ f, Rn 32.

158 Gundel, S. 82 ff.; Opermann, S. 522, Rn. 1299 f.; Hans-Joachim, Schütz, "Cassis de Dijon, EuGH - Urteil vom 20.2.1979“, JURA 1998, S. 636 ff.

${ }_{159}^{159} \mathrm{Vgl}$. Astrid Epiney, S. 63; Schiek, 1997, S. 111-112.

${ }^{160}$ Müller-Graff, $\mathrm{Rn} 196$. 


\section{ii. Stellungnahme}

Die sämtlichen Grundfreiheiten schützen gemäß der Rechtsprechung des EuGH in ihren Anwendungsbereich sowohl vor diskriminierenden als auch vor nichtdiskriminierenden Beschränkungen, sofern sie aus sachlichen Gründen nicht gerechtfertigt sind. Es handelt sich dabei um die Kollision zwischen zwei Rechtsgütern, nämlich den Rechten von Einzelnen und den Interessen der Mitgliedstaaten. Zum Ausgleich zwischen beiden Rechtsgütern sieht der EGVertrag eine Reihe von Rechtfertigungsgründen vor. Sie reichen heute aber für den Schutz berechtigter Interessen der Mitgliedstaaten nicht aus. Aus diesem Grund hat der EuGH im Wege der Rechtsfortbildung weitere Rechtfertigungsgründe ( $\mathrm{sog}$. zwingende Erfordernisse des Allgemeinwohls) entwickelt. Der EuGH hat den Anwendungsbereich dieser ungeschrie-benen Rechtfertigungsgründe zwar ursprünglich nur auf diskriminierungsfreie Beschränkungen der Grundfreiheiten beschränkt, verfolgt aber heute keine einheitliche Linie.

An dieser Stelle ist zu vermerken, daß eine unterschiedliche Rechtfertigungssystematik bezüglich der diskriminierenden Maßnahmen nicht notwendig ist. Vielmehr sollten die ungeschriebenen Rechtfertigungsgründe der zwingenden Erfordernisse des Allgemeinwohls (genauso wie die im EG-Vertrag genannten Rechtfertigungsgründe) für die sämtlichen Maßnahmen herangezogen werden. Diese Auffassung beruht nicht darauf, daß eine genaue Abgrenzung zwischen unterschiedlich und unterschiedslos anwendbaren nationalen Maßnahmen überwiegend sehr schwierig vorzunehmen ist, sondern läßt sich allein damit begründen, daß eine nationale Maßnahme, welche dem Schutz eines Allgemeininteresses dienen soll, nicht nur zu Beschränkungen, sondern auch zu Diskriminierungen führen $\mathrm{kann}^{161}$.

Hinsichtlich der Rechtfertigung eines Eingriffs ist allein der Grundsatz der Verhältnismäßigkeit von entscheidender Bedeutung. Danach ist die Rechtfertigung nationaler Maßnahmen im Falle einer (verdeckten) Diskriminierung strenger zu prüfen, als im Falle einer bloßen Beschränkung. Hinsichtlich der Rechtfertigung einer Diskriminierung ist also zusätzlich zu fragen, ob eine Differenzierung zwischen ausländischen und inländischen Marktteilnehmern zum Schutz eines Allgemeininteresses erforderlich und angemessen ist. Ein Verstoß gegen das Gemeinschaftsrecht liegt dann vor, wenn ein milderes Mittel den Zweck in gleicher Weise zu erfüllen vermag wie die zu diskriminierende staatliche Maßnahme.

${ }^{161}$ So auch Astrid Epiney, "Art 28 EGV", in: Christian Calliess/Matthias Ruffert (Hrsg.), EUV/EGV Kommentar, 1. Aufl., Luchterhand, Neuwied 1999, Rn. 38. 


\section{Zusammenfassung und Schluß}

Der EG-Vertrag sieht ein umfassendes Verbot der Diskriminierung vor, welches auf eine Unterbindung der benachteiligenden Ungleichbehandlungen aus Gründen der Herkunft aus einem anderen Mitgliedstaat abzielt. Vergleichbare Sachverhalte dürfen nicht unterschiedlich, bzw. nicht zum Nachteil von ausländischen Personen und Produkten, behandelt werden. Seine Grundlagen finden sich zum einen in Art. 12 EGV, zum anderen in den Grundfreiheiten des EG-Vertrages.

Der EuGH will jetzt alle Grundfreiheiten als Einheit verstehen. Er gibt die deutliche Tendenz zu erkennen, die Grundfreiheiten dogmatisch nach und nach anzugleichen, ohne indes eine Identität ihrer Schutzbereiche zu beabsichtigen. Die Grundfreiheiten gewährleisten nach ständiger Rechtsprechung des EuGH neben dem Diskriminierungsverbot auch das Beschränkungsverbot.

Die Diskriminierungsverbote der Grundfreiheiten umfassen genauso wie das Diskriminierungsverbot des Art. 12 EGV alle Formen der Diskriminierung. Die gemeinschaftsrechtswidrigen Diskriminierungen sind insofern leicht festzustellen, als sie formell an Unterscheidungsmerkmal von Personen, Produkten anknüpfen. In der Praxis werden die Diskriminierungen durch nationale Maßnahmen jedoch zumeist verdeckt herbeigeführt. Nationale Vorschriften sind zwar neutral, d.h. für alle Marktteilnehmer gleich formuliert, wirken sich aber wegen faktischer Unterschiede für ausländische Marktteilnehmer ungleich aus. Da verdeckte Diskriminierungen tatsächlich zu dem gleichen Ergebnis führen wie offene Diskriminierungen, müssen sie im Prinzip die Rechtsfolgen offener Diskriminierungen vorsehen.

Verdeckte Diskriminierungen sind zumeist nur schwer faßbar. Dabei kann es im Einzelfall wiederum schwierig sein, die Fälle der verdeckten Diskriminierung von denen der unterschiedslos geltenden Beschränkung abzugrenzen, was nach dem gegenwärtigen Meinungsstand auf der Rechtfertigungsstufe von Bedeutung ist. Denn die Tatbestände der beiden Eingriffe in die Grundfreiheiten gehen fließend ineinander über. Daher ist es nicht eine leicht zu lösende Aufgabe, sie in der Praxis voneinander zu unterscheiden. Die Abgrenzung von verdeckten Diskriminierungen gegenüber Beschränkungen ist nicht trennscharf möglich. In zahlreichen Urteilen, in welchen der EuGH das Beschränkungsverbot zur Anwendung brachte, handelte es sich um Fälle, die ebenso gut durch ein weit verstandenes Verbot verdeckter Dis-kriminierungen zu erfassen gewesen wären.

Die Grundfreiheiten sind jedoch nicht schrankenlos gewährleistet, sondern dürfen nach den ausdrücklichen Regelungen des EG-Vertrages unter den dort genannten Voraussetzungen von den Mitgliedstaaten beschränkt werden. 
Einigkeit herrscht ferner darüber, daß bloße Beschränkungen zusätzlich auch durch zwingende Erwägungen des Allgemeinwohls gerechtfertigt werden können, sofern das Ziel mit verhältnismäßigen Mitteln angestrebt wird. (CassisRechtsprechung). In Rechtsprechung und Literatur ist jedoch der Anwendungsbereich der zwingenden Erfordernisse des Allgemeininteresses bisher nicht eindeutig geklärt worden. Bei (formellen) Diskriminierungen schließt der EuGH im Prinzip die Rechtfertigung durch zwingende Erfordernisse des Allgemeininteresses aus. In jüngster Zeit hat er aber eine Reihe von Urteilen gefällt, die diese strikte Differenzierung zwischen diskriminierenden und unterschiedslos anwendbaren Maßnahmen nicht beachten, und die Rechtfertigung (verdeckt) diskriminierender Regelungen am Maßstab der zwingenden Erfordernisse des Allgemeinwohls geprüft. Diesem Ansatz der Rechtsprechung ist zuzustimmen, weil ein gerechtfertigter Ausgleich zwischen den Rechten von Einzelnen und den Interessen der Mitgliedstaaten nur damit erreicht werden kann.

Zum Schluß ist die folgende These aufzustellen: Die Unterscheidung zwischen den verschiedenen Kategorien der Rechtfertigungsgründe sollte ganz aufgegeben werden. Würden alle Beschränkungen einer Rechtfertigung durch das Allgemeininteresse zugänglich, könnte dem Grad der Ungleichbehandlung durch entsprechende Gewichtung in der Verhältnismäßigkeitsprüfung einzelfallgerecht berücksichtigt werden. 